\title{
Combination of Local Transcatheter Arterial Chemoembolization and Systemic Anti-Angiogenic Therapy for Unresectable Hepatocellular Carcinoma
}

\author{
Eleni Liapi Jean-Francois H. Geschwind \\ Division of Vascular and Interventional Radiology, Johns Hopkins University School of Medicine, \\ Interventional Radiology Center, Baltimore, Maryland, USA
}

\section{Key Words}

Angiogenesis · Clinical trials · Hepatocellular carcinoma ·

Transcatheter arterial chemoembolization

\begin{abstract}
The pathophysiologic complexity of hepatocellular carcinoma (HCC) and underlying hepatic cirrhosis, make optimal treatment choice a clinical challenge. The radical change in the treatment algorithm of patients with advanced unresectable HCC over the past 7 years, with the introduction of anti-angiogenic agents in patients with only preserved liver function reflect this challenge.Even though data from studies on the combination of transcatheter arterial chemoembolization and anti-angiogenic agents demonstrate a survival advantage in selected patients, this combination is not straightforward. In this review, we'll examine current data of administering anti-angiogenic therapy in combination with transcatheter arterial chemoembolization and critically evaluate the progress and gaps in current knowledge.
\end{abstract}

Copyright $@ 2012$ S. Karger AG, Basel

\section{Introduction}

The worldwide yearly incidence of hepatocellular carcinoma (HCC) is approximately 500,000 people, with virtually 20,000 new cases occurring in the United States (US) [1]. Hepatic cirrhosis, present in 80-90\% patients with HCC, influences prognosis and treatment

Jean-Francois H. Geschwind, MD

Division of Vascular and Interventional Radiology,

Johns Hopkins University School of Medicine,

Interventional Radiology Center

Sheikh Zayed Tower, Suite 7203 The Johns Hopkins Hospital 1800 Orleans

Street Baltimore, Maryland 21287 (USA)

Tel. +1 410614 6597, E-Mail: jfg@jhmi.edu 
options in these patients. Interestingly, up to $30 \%$ patients have early stage HCC and are eligible for curative therapies, including resection or transplantation. For patients with unresectable HCC, several nonsurgical palliative treatment options are available, including transcatheter arterial chemoembolization (TACE) [2]. TACE takes advantage of hepatic tumors' preferential blood supply to selectively deliver conventional chemotherapy and embolic material to the tumor vascular bed, while sparing the surrounding hepatic parenchyma [2]. TACE has been shown to have a survival benefit in patients with intermediate and advanced HCC [2-4]. Regardless of whether TACE is performed with lipiodol and chemotherapy (conventional TACE) or with doxorubicin-eluting beads (DEB-TACE), embolization of the tumorfeeding artery is a common denominator and a key step in the procedure. This crucial step may allow the chemotherapeutic drug to remain inside the tumor longer; however, it may also create a hypoxic and ischemic tumor microenvironment. Ischemia and hypoxia, in turn, may upregulate hypoxia inducible factor- $1 \alpha$ and stimulate the expression of vascular endothelial growth factor (VEGF), leading to rebound neovascularization, tumor re-growth, and progression [5-6]. This technical limitation may subsequently offset the patient's survival benefit [7].

Folkman et al first described the central role of angiogenesis in tumor progression in a classic study showing that tumors cannot grow beyond $2 \mathrm{~mm}$ without the formation of new blood vessels [8]. This complex process leads to tumor progression and eventually tumor metastasis $[8,9]$. Several factors, including tumor HIF-1 $\alpha$, VEGF, cytokines, oncogene activation, and other mutations interact to stimulate angiogenesis $[9,10]$. Therefore, targeted inhibition of angiogenesis can be achieved at any of the aforementioned levels, with treatments including neutralization of growth factors with monoclonal antibodies, inhibition of downstream signaling from tyrosine kinase receptors, and interference with interaction between proliferating endothelial cells and matrix components.

Theoretically, the tumor vasculature may undergo morphological changes following anti-angiogenic therapy. Immature blood vessels are pruned, blood vessel tortuosity and dilation decrease, and a closer association is induced between pericytes and endothelial cells [11]. As a result of these changes, vascular permeability and interstitial fluid pressure decrease. In animal tumor models, it has been demonstrated that if chemotherapy is administered following this anti-angiogenic drug-induced vascular remodeling, increased amounts of the chemotherapeutic drug will reach the tumor cells. In addition, an increased number of tumor cells will be exposed to the chemotherapeutic drug [12]. In addition, there is data suggesting that chemotherapy may have direct anti-angiogenic effects by destructing tumor endothelial cells, bone marrow-derived cells (e.g., endothelial progenitor cells), and/or circulating endothelial cells, thus indirectly assaulting cancer cells [13, 14].

Until recently, there was no standard systemic therapy for unresectable HCC. The introduction of anti-angiogenic therapies such as sorafenib, a multikinase inhibitor, have changed the management algorithm of patients with advanced disease [15]. Because anti-angiogenic drugs act on a different level than does conventional chemotherapy, combinations of conventional cytotoxic chemotherapies such as chemoembolization and anti-angiogenic drugs may be useful for maximizing therapeutic efficacy. Currently, there are nearly 50 clinical trials assessing molecular-targeting agents in combination with chemoembolization for intermediate and advanced HCC. The sequence of administration of these therapies is of ultimate importance and currently under investigation. Here we examine current data concerning the administration of anti-angiogenic drugs in combination with TACE. 


\section{Sorafenib in Combination with TACE as Treatment for Advanced Unresectable HCC}

Sorafenib(Nexavar, BayerHealthCarePharmaceuticals,Inc.,Wayne,NJ,USA) is a multikinase inhibitor that acts on vascular endothelial growth factor receptors (VEGFR-2 and VEGFR-3), platelet-derived growthfactorreceptor(PDGFR),c-KIT,andRAF signaling, thusinhibiting cancer cellgrowthandangiogenesis directly.Sorafenib wasdiscoveredalmostadecadeago whilescreening for inhibitors of RAF protein activity $[16,17]$. Two phase III trials [Sorafenib Hepatocellular Carcinoma Assessment Randomized Protocol (SHARP) and the Asia-Pacific Study] showed that sorafenib prolonged the survival of patients with advanced HCC $[15,18]$. In these studies, sorafenib broke through the six-month median survival ceiling, with selected HCC patients surviving a median of 10.7 months [15]. Sorafenib quickly received wide acceptance and was enthusiastically adopted by HCC experts worldwide. Based on the positive results of the SHARP trial, the European Commission and the Food and Drug Administration (FDA) approved sorafenib for the treatment of advanced HCC in 2007, and in Japan, in 2009 [1]. The clear success with sorafenib has prompted interest in evaluating its use alone or in combination with other established treatments for HCC, such as TACE, as well as its usefulness for the earlier stages of the disease.

\section{Timing and therapeutic schedule of sorafenib and TACE}

Strebel et al have identified and proposed that most combination treatment regimens of TACE and sorafenib fall under three categories of administration: sequential, interrupted, and continuous [19]. In the sequential schedule, patients are first treated with TACE, and sorafenib treatment is initiated once the TACE sessions are completed. In the interrupted schedule, patients are placed on sorafenib in between TACE sessions, and sorafenib is not administered during TACE. In the continuous approach, patients are treated with sorafenib without interruption before, during, and after TACE. Table 1 presents an overview of the studies discussed further for each treatment scheme.

Sequential administration of sorafenib after TACE

The rationale for administering sorafenib after TACE lies in the observation that HCC recurrence after TACE may be due to the upregulation of VEGF expression, which results in neovessel formation in residual tumors. This surge of VEGF following TACE has been reported to peak within the first 24 hours following the procedure [5]. Moreover, the subsequent administration of sorafenib following TACE may curb the further deterioration of liver function. Nevertheless, there is significant variability in the exact timing of sorafenib administration after TACE; this may explain the conflicting results of studies that were conducted using this scheme.

A double-blind, placebo-controlled, phase III trial designed before the results of the SHARP and sorafenib Asia-Pacific trials were reported was conducted in Japan and South Korea. It involved 458 patients with unresectable HCC, Child-Pugh class A cirrhosis, and $\geq 25 \%$ tumor necrosis or shrinkage 1 to 3 months after 1 or 2 TACE sessions. Almost half the patients received TACE with gelatin foam, lipiodol, and epirubicin. Patients were randomized 1:1 to receive either $400 \mathrm{mg}$ of sorafenib twice a day (bid) or placebo, and they were treated until progression/recurrence or unacceptable toxicity occurred. The primary end-point was time to radiologic progression (TTRP), and the secondary end-point was overall survival (OS). The study failed to show an advantage of sorafenib over placebo, with a median TTRP of 5.4 and 3.7 months in the sorafenib and placebo groups, respectively $(p=0.252)$. These results were attributed to the fact that $73 \%$ patients had dose reductions and $91 \%$ had dose interruptions, leading to a much lower than planned median daily dose of sorafenib (386 mg). This study 


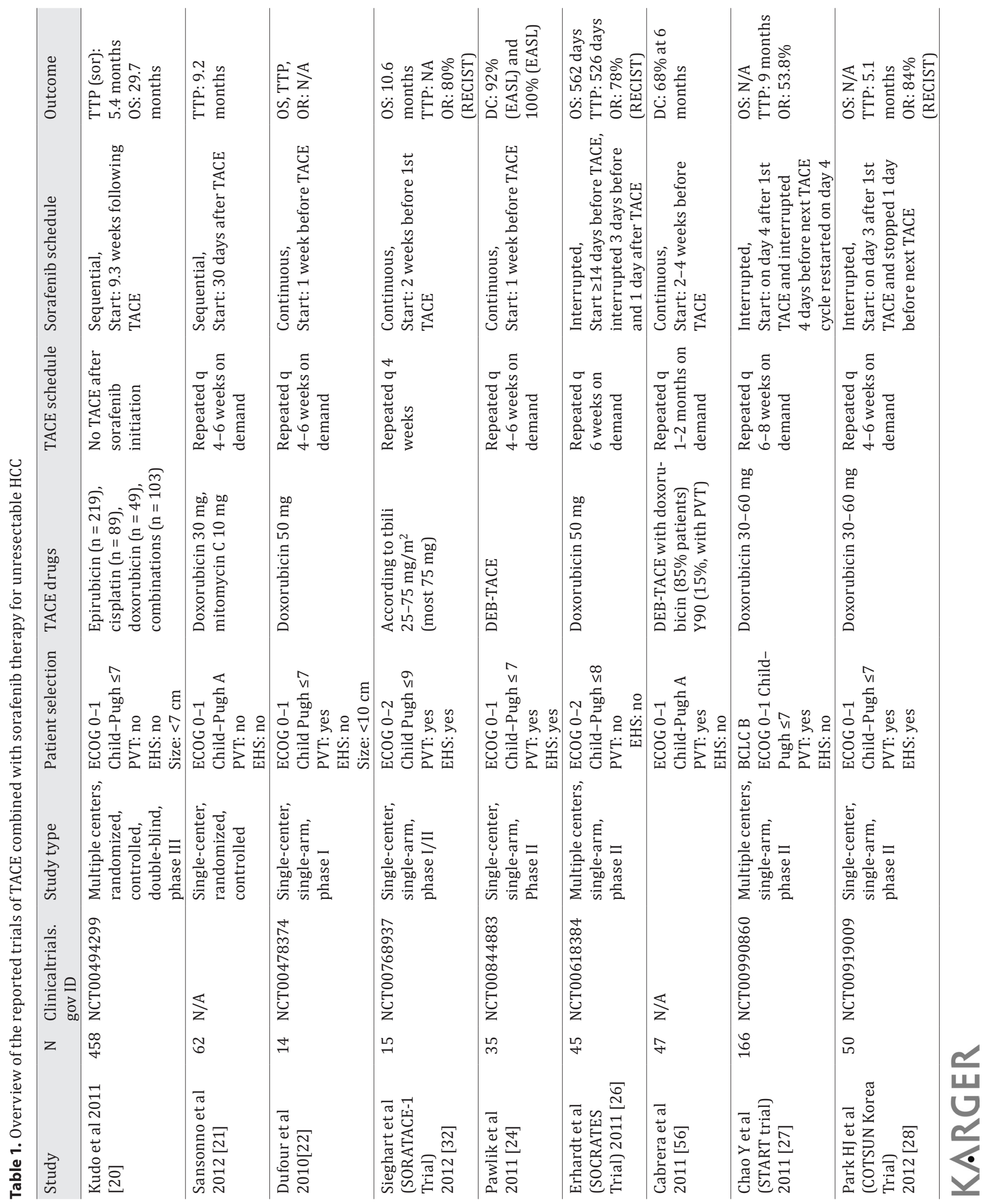


was also criticized for the long delay in starting sorafenib after TACE, with a median lag of 9.3 weeks between TACE and the start of sorafenib therapy [20]. Interestingly, the investigators chose TTRP rather than time to clinical progression as the study's primary endpoint, and progression was defined as a $\geq 25 \%$ increase in tumor size or development of a new lesion.

In another single-center, randomized study that was conducted between 2007 and 2011, 62 HCV-positive patients with Barcelona Clinic Liver Cancer (BCLC) stage B HCC received either sorafenib (400 mg bid) or placebo 30 days after TACE [21]. TACE was performed with doxorubicin (30 mg), mitomycin C (10 mg), and $10 \mathrm{ml}$ of lipiodol, followed by embolization with gelatin sponge pledgets. Endpoints were TTP and safety. The median TTP was 9.2 months in the sorafenib group and 4.9 months in the placebo group [hazard ratio (HR) $=2.5$; $95 \%$ confidence interval (CI), 1.66-7.56; $\mathrm{p}<.001$ ]. Metachronous, multicentric HCC progression occurred less frequently in sorafenib-treated patients than in placebo-treated patients $(\mathrm{p}<.05)$. Adverse reactions to sorafenib prompted the withdrawal of 9 (22\%) patients from the study.

Continuous administration of sorafenib combined with TACE for advanced HCC

The delivery of chemotherapy after anti-angiogenic drug administration may lead to increased intratumor drug delivery and an increase in the number of tumor cells that would be more sensitive to chemotherapy, presumably because of vascular changes caused by the anti-angiogenic therapy. A recent study showed improved penetration of the chemotherapeutic drug into the tumor on vessel normalization [12]. Moreover, because growth factors are secreted shortly after each TACE session, continuous treatment with sorafenib combined with TACE may be an effective approach. As with the sequential scheme, results from studies conducted using the continuous schedule of sorafenib administration are conflicting. It seems that the sorafenib starting time point, sorafenib dose adjustment, and a close multidisciplinary follow-up may play an important role in the initial steps of exploring this type of treatment schedule. A close look at the data presented for each of these studies may aid in identifying issues related to the treatment schedule.

The first published, open-label, phase I study of 14 patients treated with a continuous schedule of sorafenib (dose escalation from 200 to $400 \mathrm{mg}$ bid), starting seven days prior to TACE with doxorubicin (50 mg), showed that the continuous combination treatment regimen was tolerable and had an adverse event (AE) profile similar to that of sorafenib monotherapy [22]. The median duration of sorafenib therapy was 246 days (range, 14-547 days). After treatment with sorafenib and TACE, there was a significant decrease in the concentration of plasma VEGF. However, another phase I study from Europe, including 15 patients with primarily Child-Pugh A cirrhosis and HCC tumors of intermediate stage, reported very negative results and was stopped prematurely because of safety concerns [23]. There were 32 serious AEs (grade $\geq 3$ ) and four TACE-related deaths. Abdominal pain, weight loss, alopecia, fatigue and/or hyperbilirubinemia were observed in more than $66 \%$ cases. Patients were on sorafenib for a median duration of 5.2 months and received a median of three TACE sessions. Median OS in this study was 10.6 months.

The first US-based, single-center, single-arm, prospective, phase II study on the continuous mode of sorafenib administration combined with TACE was recently published in 2011 [24]. In this study, 35 patients with unresectable HCC (mostly BCLC-stage C, Child-Pugh score A) received DEB-TACE and sorafenib (400 mg bid). The most common toxicities reported during the first cycle were fatigue (94\%), anorexia (67\%), increase in liver enzymes (64\%), and dermatological AEs (48\%). Most patients experienced at least one grade 3/4 toxicity. During the second cycle, the number of toxicities decreased. Over the course of the study, there were 40 sorafenib dose interruptions and 25 sorafenib dose reductions. The combination of sorafenib and DEB-TACE was associated with a disease control rate of $95 \%$ according to the Response Evaluation Criteria in Solid Tumors (RECIST) criteria and 100\% according to the 
European Association for the Study of the Liver (EASL) criteria, with an objective response of $58 \%$.

In the most recent, global, phase II, randomized, double-blind, placebo-controlled SPACE study (sorafenib or placebo in combination with DEB-TACE), 307 patients with unresectable intermediate HCC and Child-Pugh A cirrhosis were randomized to receive sorafenib (400 mg bid) or placebo in a continuous scheme, followed by DEB-TACE sessions [25]. All patients received DEB-TACE (doxorubicin, $150 \mathrm{mg}$ ) 3-7 days after the first dose of the study drug, followed which they received it on day 1 ( \pm 4 days) of cycles 3,7 , and 13 , and every 6 cycles thereafter. Patients were allowed optional DEB-TACE sessions between cycles 7 and 13 and cycles 13 and 19. The primary endpoint was TTRP as evaluated by independent reviewers, and the secondary endpoints were OS, time to vascular invasion or extrahepatic spread (EHS), time to untreatable progression, and safety. A statistically significant difference was observed in TTP between sorafenib and placebo (median TTP, 169 days; HR, 0.797; $95 \%$ CI, 0.588-1.080; $\mathrm{p}=0.072$ ). There were no unexpected safety findings. Median treatment duration in the sorafenib and placebo groups was 4.8 and 6.3 months, respectively, and the median daily dose of the study drug was $566 \mathrm{mg}$ and $791 \mathrm{mg}$, respectively.

Interrupted schedule

In the interrupted schedule, administration of sorafenib is withheld for a brief time (usually 2-3 days) around the chemoembolization procedure. The advantage of this scheme is that additive toxicities caused by the combination of drugs are avoided. However, one may argue that this interruption is rather disadvantageous, similar to the sequential scheme, because subtherapeutic sorafenib levels will be achieved when the drug is most needed.

In a European phase II study of 45 patients, conducted between 2007 and 2009, sorafenib was started more than 14 days before TACE, stopped three days before TACE, and restarted one day after each TACE procedure [26]. TACE with lipiodol and $50 \mathrm{mg}$ doxorubicin was repeated at six-week intervals, if indicated. TTP was 526 days and median OS was 562 days. Grade 3 and 4 toxicities were noted in 18 and 7\% patients, respectively. There were four serious AE (SAE)-related deaths, and eight patients discontinued treatment because of AEs.

Results of the START trial (Study in Asia of the combination of TACE with Sorafenib in patients with HCC) were recently reported in 2011 [27]. In this phase II, single-arm study, 166 patients (mostly hepatitis B-positive) received TACE with doxorubicin (30-60 mg) using lipiodol and absorbable particles. Sorafenib ( $400 \mathrm{mg}$ bid) was stopped three days before and after TACE. TACE was performed on demand every 6-8 weeks, and patients that were not candidates to receive further TACE continued on sorafenib monotherapy until disease progression or unacceptable toxicity occurred. The primary objective was safety and tolerability and the secondary outcomes were TTP and OS. The mean dose of sorafenib was $743.5 \mathrm{mg}$. The median TTP was nine months, and the median OS had not been reached at the time the study results were presented. The objective response rate was $53.8 \%$, while $23.9 \%$ patients experienced grade 3 AEs, most of which were common hand-foot skin reactions (6.8\%), neutropenia (6.1\%), thrombocytopenia (3.4\%), and ALT elevation (2\%). Only 15\% patients discontinued treatment because of AEs.

In a prospective, single-arm, phase II study that was conducted in South Korea (COTSUN Trial) between 2009 and 2011, 50 BCLC B (82\% of patients) and C patients were treated with sorafenib three days after TACE and for up to 24 weeks [28]. According to the study design, repeated TACE was allowed and performed on demand. Sorafenib was withheld for one day before each TACE session and reintroduced three days after the procedure or further delayed in cases with blood abnormalities. TACE was performed with 20-60 mg of doxorubicin mixed with $2-20 \mathrm{ml}$ of ethiodized oil, followed by embolization with Gelfoam ${ }^{\circledR}$ particles. The primary objectives of this study were safety and TTP. A total of 30 patients 
completed the study, and more than half discontinued sorafenib because of disease progression. The overall median TTP was 7.1 months (95\% CI, 4.8-7.5 months), while the median TTP was 7.3 months for BCLC stage B patients and 5.0 months for BCLC stage C patients. The six-month progression-free survival (PFS) rate was 52\% (95\% CI, 37.3-66.1). The median dose intensity of sorafenib was $68.7 \%$ (range, 37.3-100) of $800 \mathrm{mg}$ daily. The most common reasons for dose reduction were hand-foot syndrome and thrombocytopenia. This study has been criticized for depleting sorafenib when it is most needed, that is, within 24 hours following TACE [29].

Unlike the continuous and sequential schedules, there is currently no randomized data available on the interrupted schedule. However, the TACTICS trial, a randomized phase III study (TACTICS trial, Clinicaltrials.gov Identifier: NCT01217034) that is currently underway, may help in clarifying the efficacy of this schedule. In this trial that is being conducted by the Japan Liver Oncology Group, sorafenib is interrupted two days before TACE and resumed three days (and up to 21 days) following TACE. Interestingly, patients in this study receive an initially low dose of sorafenib (400 mg every other day), and after confirming tolerability at one week, patients resume with the standard dose of $400 \mathrm{mg}$ bid. The primary endpoint of this study is time to untreatable progression, as defined by the presence of Child-Pugh C cirrhosis, increase in tumor size by $125 \%$ from baseline, vascular invasion, and/or extrahepatic spread of more than $10 \mathrm{~mm}$.

The Eastern Cooperative Oncology Group (ECOG) E1208 is another ongoing, randomized, double-blind, prospective, multicenter, phase III study, evaluating the combination of TACE and sorafenib administered via an interrupted schedule (ClinicalTrials.gov registration number: NCT01004978). Prerandomization stratification will be performed according to ChildPugh score (A versus B7), type of TACE (conventional versus DEB-TACE), and presence or absence of macrovascular intrahepatic portal vein invasion. A maximum number of four TACE sessions within six months is allowed, after which patients will be on sorafenib maintenance until disease progression. Patients will start receiving sorafenib 10-14 days prior to TACE, so as to establish the dosage and monitor toxicity. Sorafenib will be held for 24-48 hours before TACE and will be resumed 7-14 days after each TACE session if certain criteria are met (toxicities: serum bilirubin <grade 2 , serum albumin < grade 3 , hematologic toxicity <grade 2 , and ECOG performance status $<1$ ). The primary objective of this study is PFS with TACE alone or with sorafenib. Secondary objectives include OS and toxicity, extrahepatic versus intrahepatic patterns of failure, and pharmacogenetic and pharmacokinetic properties of sorafenib.

\section{Current issues with combined TACE and sorafenib treatment schemes}

A recent phase I study on the pharmacokinetics of sorafenib showed that patients with moderate to severe hepatic and renal dysfunction cannot tolerate the dose of $400 \mathrm{mg}$ bid, which is recommended for patients with normal organ function [30]. In this study, patients with total bilirubin $>1.5$ times the upper normal limit and any AST value could not tolerate the recommended 400-mg bid sorafenib prescription. This observation may suggest that total bilirubin may be the most important clinical marker of subsequent sorafenib tolerability. Interestingly, a recent study investigated whether patients carrying UGT1A1*28 and treated with sorafenib had increased sorafenib exposure or an increased risk of developing hyperbilirubinemia [11]. The clinical data from this study suggest that sorafenib can cause hyperbilirubinemia in patients with Gilbert's syndrome, which can lead to abnormally high or low sorafenib exposure.

Absorption pharmacokinetics in patients with advanced HCC who received a single 400mg dose of sorafenib or multiple doses of $400-\mathrm{mg}$ bid sorafenib have been detailed in two studies [30]. Differences in pharmacokinetics between Child-Pugh A and B patients were not 
considered clinically significant in either trial. However, few studies are currently enrolling patients having cirrhosis with a Child-Pugh B score of $>7$.

TACE most commonly involves the administration of doxorubicin. The concomitant administration of doxorubicin and sorafenib influences the pharmacokinetics of doxorubicin, with more than a $20 \%$ increase in the area under the curve for doxorubicin concentration $[31,32]$. This is essential for calculating the lifetime cumulative dosage of doxorubicin and the development of symptomatic congestive heart failure.

Recent studies suggest that tumor progression following treatment with sorafenib may result in either evasive or intrinsic resistance [33]. Our limited understanding of the mechanism of action of sorafenib in advanced HCC, however, makes it even more difficult to determine the possible resistance mechanisms. Preclinical models suggest that phenotypic resistance to VEGFR inhibition can induce the activation of VEGF-independent angiogenic signals by members of the fibroblast growth factor (FGF) family. One way to overcome this resistance is to combine treatments that indirectly and/or directly inhibit angiogenesis [34].

Currently, most studies are designed to stop evaluating patients on appearance of radiological progression. However, data from the SHARP trial suggest that there is evidence that patients survive longer even though they quickly progress radiologically. Several questions may arise with regard to patient management in such scenarios where liver function remains stable but cancer progresses radiologically. For example, is an increase in sorafenib dose to $600 \mathrm{mg}$ bid justified? Should sorafenib be discontinued? Should TACE be discontinued? Should the combination therapy be further continued? Should other anti-angiogenic therapies be considered? If yes, with or without TACE?

\section{Bevacizumab and TACE}

One of the major pathways involved in the process of angiogenesis is via the VEGF family of proteins and receptors. VEGF is an endothelial cell mitogen that regulates proliferation, permeability, and survival of endothelial cells through inhibition of apoptosis $[13,35$, 36]. Because of its fundamental role in tumor angiogenesis, the VEGF/VEGFR pathway was recognized very early as a potential anti-cancer target, and it has been a major focus of research and development in the field of oncology over the past decade [14, 35]. Therefore, it is not surprising that the first anti-angiogenic treatment strategies focused on inhibition of the VEGF pathway. HCC is one of the most vascular solid cancers, associated with a high tendency for vascular invasion and a high VEGF expression [3, 37, 38]. Upregulation of VEGF has been correlated with increased tumor invasion, intratumoral microvessel density, disease recurrence, and poor prognoses [39-41]. Bevacizumab is a humanized monoclonal antibody that binds VEGF and inhibits the interaction of VEGF with its receptors on the surface of endothelial cells. When uninhibited, this interaction may lead to endothelial cell proliferation and new blood vessel formation [42]. VEGF expression is known to play an important role in HCC development, and the degree of its expression is reported to be associated with tumor size and histological grade. Bevacizumab was the first anti-angiogenic drug to be tested in combination with TACE for patients with unresectable HCC. Nevertheless, the mechanisms of the interplay of bevacizumab with TACE have not been fully explored. One possible theory is that bevacizumab, by normalizing tumor vessels, may also normalize intratumoral pressure and increase tumor oxygenation, thus making HCC tumors more vulnerable to the chemotherapy of TACE. The combination of TACE and bevacizumab, however, has not been tested as extensively as others primarily because of safety concerns, particularly bleeding risk, which was reported early on in several small studies [43]. Moreover, unlike sorafenib, 
which induces tumor regression in HCC by inhibiting angiogenesis as well as other signaltransduction pathways, bevacizumab works only via angiogenic inhibition.

The results of the first US-based, single-arm, phase II study aiming to investigate the role of anti-angiogenic therapy prior to TACE were presented in 2010 (trial registration: clinicaltrials.gov NCT00335829) [44]. The study evaluated safety and tumor response to therapy in 26 patients with unresectable HCC (ECOG status 0-2, Child-Pugh stage A-B, BCLC stage A-C). These patients received $10 \mathrm{mg} / \mathrm{kg}$ of bevacizumab every two weeks in addition to TACE in a six-week cycle (average 1-3 cycles). The primary endpoint was tumor response as assessed by magnetic resonance imaging (MRI) at baseline and three weeks after TACE, size (RECIST), and contrast-enhancement (EASL). Secondary endpoints included safety and survival. On follow-up imaging, index lesions had a mean decrease in size of $13 \%(\mathrm{p}<0.0005)$. Eight $(35 \%)$ achieved partial response and $15(65 \%)$ had stable disease as per RECIST. Targeted tumors demonstrated a mean decrease in contrast enhancement of $69 \%(\mathrm{p}<0.0005)$. As per EASL criteria, $14(60 \%)$ patients had complete or partial response and nine (39\%) had stable disease. The disease control rate was $100 \%$ by either criterion. Median OS was 13.5 months, with 10 survivors at the end of the study. Overall, the combination therapy with bevacizumab and TACE was reasonably well tolerated. A total of 15 (58\%) patients experienced grade 3/4 toxicities that were possibly related to either therapy, with $38 \%$ toxicities eventually resolving. The 30 -day mortality rate in this study was $4 \%$.

In another recent US-based, single-center, randomized, controlled, phase II study (trial registration: clinicaltrials.gov NCT00049322), 30 patients with intermediate HCC undergoing TACE were randomized into two groups, with the treatment arm receiving $10 \mathrm{mg} / \mathrm{kg}$ of bevacizumab starting one week prior to TACE and the control arm remaining under clinical observation without any drug [6]. TACE was performed at week 1 and at week 16, after which patients were allowed to cross over. Additional angiographies without TACE were performed on weeks 10 and 14. The primary endpoint of this study was quantification of neoangiogenesis by angiography, and the secondary endpoints were PFS, OS, and VEGF levels before and after TACE with and without bevacizumab. Neovessels formed less frequently in the treatment arm than in the observational arm (14 and 33\%, respectively), but this difference was not statistically significant. PFS at 16 weeks was $79 \%$ in the treatment arm and $19 \%$ in the observation arm $(\mathrm{p}=0.021)$. The median OS was 61 months in the treatment arm and 49 months in the observation arm $(\mathrm{p}=0.21$, intention-to-treat analysis, independent of patient crossover). Interestingly, 12 patients underwent transplantation after completing the study. No life-threatening, bevacizumab-related toxicities were observed in this study.

A single-center, prospective, double-blind, randomized, controlled phase II study of patients with unresectable HCC treated with TACE and bevacizumab or TACE and placebo (AVATACE-1 trial) was terminated prematurely because of safety concerns in the treatment arm (trial registration: clinicaltrials.gov NCT00280007) [45]. Patients in the treatment arm were assigned to receive $5 \mathrm{mg} / \mathrm{kg}$ of intravenous bevacizumab every 14 days for 52 weeks in addition to standard TACE. The primary endpoints of this study were effectiveness of bevacizumab in combination with TACE, as determined by the absence of tumor progression after a maximum of one year of treatment with bevacizumab and by the assessment of collateral tumor vessel growth on MRI/computed tomography. A total of 27 patients with primarily BCLC stage B HCC and Child-Pugh A cirrhosis were randomized to receive bevacizumab (13 patients) or placebo. Most patients received an average of three TACE sessions within the study time frame ( 52 weeks). The study investigators observed the following grade 4 and 5 toxicities in the bevacizumab arm: severe vascular and bleeding-related AEs (grade 4 and 5) in five patients $(18.5 \%)$, myocardial infarction in two, esophageal variceal bleedings with fatal outcomes in two, arterial occlusion of the lower leg in one, portal vein thromboses (PVT) in three, and femoral artery aneurysm in one patient. Furthermore, bacterial infections leading 
to sepsis from different causes were only seen in the bevacizumab group (46\% of patients in this group).

All these trials were single-arm trials, precluding any conclusions about efficacy. We are awaiting the results of only one more phase II, single-arm, open-label study that has tested this combination (5 mg/kg of bevacizumab; trial registration: clinicaltrials.gov NCT00576199); therefore, it is unlikely that other investigators will further explore the combination of TACE and bevacizumab alone. However, in the era of HCC resistance to bevacizumab or sorafenib, research on combinations of other anti-angiogenic drugs and chemotherapy, possibly that including TACE, is attracting much interest.

\section{Combination of New Anti-angiogenic Drugs Targeting Other Pathways}

\section{Combination of brivanib and TACE}

FGF is overexpressed and activated in HCC. High FGF-2 levels may correlate with a poor clinical outcome in patients with advanced HCC. Brivanib alaninate (BMS-582664, Bristol-Myers Squibb, NY, NY, USA) is an oral, small-molecule, tyrosine kinase inhibitor that selectively blocks FGF and VEGF signaling. Brivanib has shown potent antitumor activity in preclinical models of HCC [46] and has demonstrated significant antitumor activity and acceptable tolerability in a phase II, open-label study of patients with unresectable, locally advanced or metastatic HCC. In this trial, brivanib showed anti-tumor activity, both as a firstline therapy, achieving an overall survival of 10 months and as second-line therapy in patients who had failed prior anti-angiogenic treatment, primarily with sorafenib, achieving an overall survival of 9.5 months. Of note, the incidence of all-grade hand-foot syndrome was only $8 \%$ in this study.

A randomized, double-blind, multicenter, phase III study of brivanib versus placebo as adjuvant therapy to TACE in patients with unresectable HCC (the BRISK TA study, ClinicalTrials.gov Identifier: NCT00908752) is currently recruiting patients [47]. The primary endpoint of this study is OS, and secondary endpoints include time to disease progression and time to extrahepatic spread or vascular invasion. Investigators aim to recruit 870 patients worldwide, with eligible patients having HCC and Child-Pugh A or B (score 7) cirrhosis. Patients will receive brivanib $200 \mathrm{mg}$ once daily until disease progression or toxicity.

\section{Combination of sunitinib and TACE}

Sunitinib (SU011248, Sutent, Pfizer Inc., NY, NY, USA) is an oral tyrosine kinase inhibitor of VEGFR, PDGFR, Flt-3, and c-KIT [16]. An initial phase II study showed a response rate of $2.9 \%$ and an average OS of 9.8 months in 34 advanced HCC patients [48]. A phase III sunitinib versus sorafenib study was, nevertheless, discontinued on the basis of a higher incidence of SAEs in the sunitinib group compared with that in the sorafenib group and the fact that sunitinib did not meet the criteria of superiority over sorafenib in terms of survival [49]. A variable dose regimen may show an improved toxicity profile and is currently being investigated in an open-label sunitinib continuation protocol involving patients who have completed a prior sunitinib study and are judged by the investigator to have the potential to benefit from sunitinib treatment (ClinicalTrials.gov Identifier:NCT00428220. Sunitinib is also being investigated in combination with TACE. A double-blind, randomized, phase II/ III study comparing chemoembolization combined with sunitinib with chemoembolization combined with placebo in patients with HCC (SATURNE) is currently recruiting patients (ClinicalTrials.gov Identifier: NCT01164202). Patients in the treatment arm will receive oral sunitinib malate once daily on days $1-28$, followed by an intermission of 7-10 days, after 


\section{Liver}

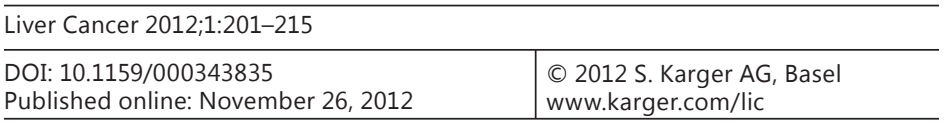

which patients will undergo TACE. The treatment is repeated every six weeks for one year. Patients are stratified according to main tumor diameter $(<5 \mathrm{~cm}$ versus $\geq 5 \mathrm{~cm})$, nodular involvement (uninodular versus multinodular), and center.

\section{Combination of TSU-68 and TACE}

TSU-68 is an oral agent that inhibits VEGFR, PDGFR, and FGFR. It recently showed promising efficacy with a high safety profile, even in heavily pretreated HCC patients with ChildPugh B liver cirrhosis. An open-label, multicenter, randomized, phase II trial of 101 patients without portal vein thrombosis or extrahepatic metastases and with either Child-Pugh A or B cirrhosis were treated with either TSU-68 $400 \mathrm{mg} /$ daily or best supportive care after TACE. TSU-68 was well tolerated after TACE. Median PFS was 5.2 months in patients treated with TSU-68 and 4.0 months in patients that received best supportive care (HR in group A, 0.699; $p=0.054)$. The most frequent grade $3 / 4$ events were fatigue (6\%), ALT elevation (26\%), AST elevation (46\%), total bilirubin elevation (6\%), hypokalemia (14\%), r-GTP elevation (8\%), lymphopenia (12\%), decreased platelet count (12\%), and ALP elevation (8\%) [50].

\section{Combination of rapalogs and TACE}

Recent data support an important role for the phosphoinositide 3-kinase (PI3 K)/Akt/ mammalian target of rapamycin (mTOR) pathway in HCC formation and potential for mTOR inhibition as a therapeutic strategy against HCC. In 2009, everolimus (RAD001, Afinitor, Novartis Pharmaceuticals, Basel, Switzerland) received initial FDA approval for the treatment of patients with advanced renal cell carcinoma, whose disease had progressed following treatment with VEGF-targeted therapies. The safety and efficacy of everolimus was initially evaluated in a phase I/II study of patients with advanced HCC who received either 5 or $10 \mathrm{mg} /$ day orally (six weeks/cycle) for 24 weeks. The 28 patients who received $5 \mathrm{mg} /$ day had a median PFS of 3.8 months and an OS of 8.4 months. The estimated PFS rate at 24 weeks was $28.6 \%$ (95\% CI, 7.9-49.3\%). On the basis of these results, the $10 \mathrm{mg} /$ day schedule was never tested $[51,52]$. Everolimus is currently being tested in a phase II, randomized, double-blind, multicenter Asian Study of localized unresectable HCC treated with or without TACE (The TRACER Study, ClinicalTrials.gov Identifier: NCT01379521). Interestingly, everolimus lowered circulating VEGF levels in contrast to sorafenib, which increased them.

\section{Combination of TACE and axitinib}

Axitinib (AG-013736, Inlyta, Pfizer Inc., NY, NY, USA) is a small-molecule, tyrosine kinase inhibitor that inhibits multiple targets such as VEGFR-1, VEGFR-2, VEGFR-3, PDGFR, and cKIT [53]. It is currently FDA approved for patients with renal cell carcinoma who fail to respond to primary treatment [53]. In a preclinical study, rapid response to axitinib has been observed within only 24 hours of treatment, with loss of endothelial sprouts and fenestration in $80 \%$ of tumor vasculature [9]. However, these vascular changes may reverse within seven days on cessation of treatment, indicating the transient and reversible nature of target inhibition [54]. In patients with advanced unresectable renal cell carcinoma, the incidence of hypertension, dysphonia, and hypothyroidism is more frequent with axitinib than with sorafenib, whereas the incidence of hand-foot syndrome, rash, and alopecia are less frequent. Grade 3 or higher toxicities, which are quite uncommon, include thromboembolic events, hemorrhage, gastrointestinal perforation, reversible posterior leukoencephalopathy syndrome, and hypothyroidism. Most AEs are manageable, resulting in a lower rate of treatment discontinuation.

A phase II study of TACE and axitinib for the treatment of unresectable HCC from Hong Kong (ClinicalTrials.gov identifier: NCT01352728) will assess efficacy of this combination. The primary endpoint is two-year survival rate, and the secondary endpoints include objective response according to RECIST, disease control rate, duration of response, PFS, OS, safety, 
quality of life, and collection of tissue biomarkers. Patients will receive axitinib $5 \mathrm{mg}$ bid for six cycles concurrently with TACE, following which they will continue with axitinib until disease progression.

\section{Current Issues in The Evaluation of Treatment Outcomes of The Combination of Anti-angiogenic Agents and TACE}

\section{Validated radiological evaluation of treatment outcomes}

Currently, there is no clear evidence with regard to the best way of assessing radiological response to treatment with a combination of TACE and anti-angiogenic agents. This is because the mechanism of action of anti-angiogenic agents and TACE is inconsistent with the assessment of response by RECIST. Even though the EASL guidelines recommend that assessment of tumor response should incorporate the decrease in viable tumor burden, these guidelines need to be further validated. Moreover, whether the current imaging techniques allow adequate and consistent quantification of tumor necrosis, or whether this quantification is a meaningful endpoint for evaluating the combination of TACE and anti-angiogenic agents for HCC treatment remains unclear.

Interestingly, in the SHARP trial, patients with or without little objective response lived longer, dispelling the notion that radiological response or radiologic TTP can be used as a surrogate marker of OS. This should warn investigators of the need to conceptually change the process of patient evaluation and outcome assessment when molecular-targeted therapies are involved in liver cancer treatments.

\section{Validated serum biomarkers}

A very recent study from the SHARP population investigated whether 10 biomarkers (baseline concentrations and changes over 12 weeks) could predict patient prognosis or response to treatment [55]. The concentrations of two biomarkers, VEGF and Ang2, predicted patient survival, suggesting that both may be included in prognostic staging systems for patients with HCC. In addition, concentrations of soluble c-KIT and hepatocyte growth factor showed a similar trend of predicting response to therapy without reaching statistical significance. However, reliable markers to monitor the activity of anti-angiogenic drugs are yet to be discovered.

\section{Conclusion}

The pathophysiological complexity of HCC and underlying hepatic cirrhosis make optimal treatment choice a clinical challenge. This problem is reflected well by the radical change in the treatment algorithm of unresectable HCC over the past five years. Even though data from recent studies on the combination of TACE and anti-angiogenic agents demonstrate a survival advantage in patients with preserved liver function, results from this combination therapy is not straightforward. Further preclinical and clinical research is therefore required. While a better understanding of disease biology is crucial, it is also imperative to further investigate the mechanisms by which anti-angiogenic agents work in concert with conventional chemotherapeutic agents used in TACE. Once these essential steps are taken, optimal planning of the timing and sequence of administration of this combination treatment can be achieved with subsequent improvements in efficacy and effectiveness. 


\section{References}

1 El-Serag HB: Hepatocellular Carcinoma. N Engl J Med 2011;365:1118-1127.

2 Kamel IR, Reyes DK, Liapi E, Bluemke DA, Geschwind JF: Functional MR imaging assessment of tumor response after 90Y microsphere treatment in patients with unresectable hepatocellular carcinoma. J Vasc Interv Radiol 2007;18:49-56.

3 Lo CM, Ngan H, Tso WK, Liu CL, Lam CM, Poon RT, et al: Randomized controlled trial of transarterial lipiodol chemoembolization for unresectable hepatocellular carcinoma. Hepatology 2002;35:1164-1171.

4 Bruix J, Llovet JM, Castells A, Montana X, Bru C, Ayuso MC, et al: Transarterial embolization versus symptomatic treatment in patients with advanced hepatocellular carcinoma: results of a randomized, controlled trial in a single institution. Hepatology 1998;27:1578-1583.

5 Li X, Feng GS, Zheng CS, Zhuo CK, Liu X: Expression of plasma vascular endothelial growth factor in patients with hepatocellular carcinoma and effect of transcatheter arterial chemoembolization therapy on plasma vascular endothelial growth factor level. World J Gastroenterol 2004;10:2878-2882.

6 Britten CD, Gomes AS, Wainberg ZA, Elashoff D, Amado R, Xin Y, et al: Transarterial chemoembolization plus or minus intravenous bevacizumab in the treatment of hepatocellular cancer: a pilot study. BMC Cancer 2012;12:16.

7 Sergio A, Cristofori C, Cardin R, Pivetta G, Ragazzi R, Baldan A, et al: Transcatheter arterial chemoembolization (TACE) in hepatocellular carcinoma (HCC): the role of angiogenesis and invasiveness. Am J Gastroenterol 2008;103:914-921.

8 Hlatky L, Hahnfeldt P, Folkman J: Clinical Application of Antiangiogenic Therapy: Microvessel Density, What It Does and Doesn't Tell Us. J Natl Cancer Inst. 2002 June 19, 2002;94:883-893.

9 Inai T, Mancuso M, Hashizume H, Baffert F, Haskell A, Baluk P, et al: Inhibition of vascular endothelial growth factor (VEGF) signaling in cancer causes loss of endothelial fenestrations, regression of tumor vessels, and appearance of basement membrane ghosts. Am J Pathol 2004;165:35-52.

10 Bergers G, Benjamin LE: Tumorigenesis and the angiogenic switch. Nat Rev Cancer 2003;3:401-410.

11 Peer CJ, Sissung TM, Kim A, Jain L, Woo S, Gardner ER, et al: Sorafenib is an inhibitor of UGT1A1 but is metabolized by UGT1A9: implications of genetic variants on pharmacokinetics and hyperbilirubinemia. Clinical cancer research: an official journal of the American Association for Cancer Research 2012;18:20992107.

12 Escorcia FE, Henke E, McDevitt MR, Villa CH, Smith-Jones P, Blasberg RG, et al: Selective Killing of Tumor Neovasculature Paradoxically Improves Chemotherapy Delivery to Tumors. Cancer Research 2010 70:9277-9286.

13 Hicklin DJ, Ellis LM: Role of the vascular endothelial growth factor pathway in tumor growth and angiogenesis. J Clin Oncol 2005;23:1011-1027.

14 Kerbel RS, Kamen BA: The anti-angiogenic basis of metronomic chemotherapy. Nat Rev Cancer 2004;4:423436.

15 Llovet JM, Ricci S, Mazzaferro V, Hilgard P, Gane E, Blanc JF, et al: Sorafenib in advanced hepatocellular carcinoma. N Engl J Med 2008;359:378-390.

16 Kudo M, Ueshima K: Positioning of a molecular-targeted agent, sorafenib, in the treatment algorithm for hepatocellular carcinoma and implication of many complete remission cases in Japan. Oncology 2010;78(Suppl 1):154-166.

17 Wilhelm SM, Carter C, Tang L, Wilkie D, McNabola A, Rong H, et al: BAY 43-9006 exhibits broad spectrum oral antitumor activity and targets the RAF/MEK/ERK pathway and receptor tyrosine kinases involved in tumor progression and angiogenesis. Cancer Res 2004;64:7099-7109.

18 Cheng AL, Kang YK, Chen Z, Tsao CJ, Qin S, Kim JS, et al: Efficacy and safety of sorafenib in patients in the Asia-Pacific region with advanced hepatocellular carcinoma: a phase III randomised, double-blind, placebo-controlled trial. Lancet Oncol 2009;10:25-34.

19 Strebel BM, Dufour JF: Combined approach to hepatocellular carcinoma: a new treatment concept for nonresectable disease. Expert Rev Anticancer Ther 2008;8:1743-1749.

20 Kudo M, Imanaka K, Chida N, Nakachi K, Tak WY, Takayama T, et al: Phase III study of sorafenib after transarterial chemoembolisation in Japanese and Korean patients with unresectable hepatocellular carcinoma. Eur J Cancer 2011;47:2117-2127.

21 Sansonno D, Lauletta G, Russi S, Conteduca V, Sansonno L, Dammacco F: Transarterial Chemoembolization Plus Sorafenib: A Sequential Therapeutic Scheme for HCV-Related Intermediate-Stage Hepatocellular Carcinoma: A Randomized Clinical Trial. Oncologist 2012;17:359-366.

22 Dufour JF, Hoppe H, Heim MH, Helbling B, Maurhofer O, Szucs-Farkas Z, et al: Continuous administration of sorafenib in combination with transarterial chemoembolization in patients with hepatocellular carcinoma: results of a phase I study. Oncologist 2010;15:1198-1204.

23 Sieghart W, Pinter M, Reisegger M, Muller C, Ba-Ssalamah A, Lammer J, et al: Conventional transarterial chemoembolisation in combination with sorafenib for patients with hepatocellular carcinoma: a pilot study. Eur Radiol 2012;22:1214-1223.

24 Pawlik TM, Reyes DK, Cosgrove D, Kamel IR, Bhagat N, Geschwind JF: Phase II trial of sorafenib combined with concurrent transarterial chemoembolization with drug-eluting beads for hepatocellular carcinoma. J Clin Oncol 2011;29:3960-3967. 
25 Bruix RL: Sorafenib or placebo in combination with transarterial chemoembolization (TACE) with doxorubicin-eluting beads (DEBDOX) for intermediate-stage hepatocellular carcinoma (HCC): Phase II, randomised, double-blind SPACE trial. ASCO Gastrointestinal Cancers Symposium; 2012: J Clin Oncol; 2012;p. abstr LBA154.

26 Erhardt AK, FT, Dollinger MM, Schott E, Wege H, Bitzer M, Gog C, Raedle J, Schuchmann M, Walter C,Blondin D, Ohmann C, Haeussinger D; TACE plus sorafenib for the treatment of hepatocellular carcinoma: Final results of the multicenter SOCRATES trial. J Clini Oncol. 2011;29(suppl):abstr 4107.

27 Chao YL, H-C, Lee T-Y, Yoon J, Han G, Yang J, Wang J, Kim B, Shao G, Chung Y-H: START (Study in Asia of the combination of TACE (transcatheter arterial chemoembolization) with Sorafenib in patients with Hepatocellular Carcinoma) Trial. ILCA Annual conference 2011; p. 17.

28 Park JW, Koh YH, Kim HB, Kim HY, An S, Choi JI, et al: Phase II study of concurrent transarterial chemoembolization and sorafenib in patients with unresectable hepatocellular carcinoma. J Hepatol. 2012 Feb 4.

29 Dufour JF: TACE with or without systemic therapy? J Hepatol 2012;56:1224-1225.

30 Miller AA, Murry DJ, Owzar K, Hollis DR, Kennedy EB, Abou-Alfa G, et al: Phase I and pharmacokinetic study of sorafenib in patients with hepatic or renal dysfunction: CALGB 60301. J Clin Oncol 2009;27:18001805.

31 Richly H, Schultheis B, Adamietz IA, Kupsch P, Grubert M, Hilger RA, et al: Combination of sorafenib and doxorubicin in patients with advanced hepatocellular carcinoma: results from a phase I extension trial. Eur J Cancer 2009.45:579-587.

32 Sieghart W, Pinter M, Reisegger M, Muller C, Ba-Ssalamah A, Lammer J, et al: Conventional transarterial chemoembolisation in combination with sorafenib for patients with hepatocellular carcinoma: a pilot study. European Radiology. 2012 Jan 4.

33 Villanueva A, Llovet JM: Second-line therapies in hepatocellular carcinoma: emergence of resistance to sorafenib. Clin Cancer Res 2012;18:1824-1826.

34 Moreno Garcia V, Basu B, Molife LR, Kaye SB: Combining antiangiogenics to overcome resistance: rationale and clinical experience. Clinical Cancer Research: an official journal of the American Association for Cancer Research. 2012 Jul 15;18:3750-3761.

35 Dvorak HF, Brown LF, Detmar M, Dvorak AM: Vascular permeability factor/vascular endothelial growth factor, microvascular hyperpermeability, and angiogenesis. Am J Pathol 1995;146:1029-1039.

36 Ferrara N, Hillan KJ, Novotny W: Bevacizumab (Avastin), a humanized anti-VEGF monoclonal antibody for cancer therapy. Biochem Biophys Res Commun 2005.333:328-335.

37 Schneider BP, Miller KD: Angiogenesis of Breast Cancer. J Clin Oncol. 2005 March 10, 2005;23:1782-1790.

38 Jeng KS, Sheen IS, Wang YC, Gu SL, Chu CM, Shih SC, et al: Prognostic significance of preoperative circulating vascular endothelial growth factor messenger RNA expression in resectable hepatocellular carcinoma: a prospective study. World J Gastroenterol 2004;10:643-648.

39 Beecken W-DC, Fernandez A, Joussen AM, Achilles E-G, Flynn E, Lo K-M, et al: Effect of Antiangiogenic Therapy on Slowly Growing, Poorly Vascularized Tumors in Mice. J Natl Cancer Inst 2001;93:382-387.

40 Yao DF, Wu XH, Zhu Y, Shi GS, Dong ZZ, Yao DB, et al: Quantitative analysis of vascular endothelial growth factor, microvascular density and their clinicopathologic features in human hepatocellular carcinoma. Hepatobiliary Pancreat Dis Int 2005;4:220-226.

41 Takahashi Y, Kitadai Y, Bucana CD, Cleary KR, Ellis LM: Expression of vascular endothelial growth factor and its receptor, KDR, correlates with vascularity, metastasis, and proliferation of human colon cancer. Cancer Res 1995;55:3964-3968.

42 Kim KJ, Li B, Winer J, Armanini M, Gillett N, Phillips HS, et al: Inhibition of vascular endothelial growth factor-induced angiogenesis suppresses tumour growth in vivo. Nature 1993;362:841-844.

43 Siegel AB, Cohen EI, Ocean A, Lehrer D, Goldenberg A, Knox JJ, Chen H, Clark-Garvey S, Weinberg A, Mandeli J et al:Phase II trial evaluating the clinical and biologic effects of bevacizumab in unresectable hepatocellular carcinoma. J Clin Oncol 2008;26:2992-2998.

44 Reyes DK, Vossen JA, Kamel IR, Mulcahy MF, Salem R, Messersmith WA, et al: Phase II trial of bevacizumab combined with transarterial chemoembolization (TACE) for hepatocellular carcinoma: Initial experience at two institutions. ASCO Gastrointestinal Cancers Symposium 2010; Orlando, FL; 2010.

45 Ulbrich GP M, Reiberger T, Ferlitsch A, Sieghart W, Peck-Radosavljevic M: Interim safety-analysis of the AVATACE-1 trial (Bevacizumab as inhibitor of collateral growth during transarterial chemoembolization (TACE) for hepatocellular carcinoma- a double-blind randomized placebo-controlled pilot-trial. Journal of Hepatology 2010;55(Supplement 1).

46 Huynh H, Ngo VC, Fargnoli J, Ayers M, Soo KC, Koong HN, et al: Brivanib alaninate, a dual inhibitor of vascular endothelial growth factor receptor and fibroblast grow th factor receptor tyrosine kinases, induces growth inhibition in mouse models of human hepatocellular carcinoma. Clinical Cancer Research: an official journal of the American Association for Cancer Research 2008;14:6146-6153.

47 Kudo M: Future treatment option for hepatocellular carcinoma: a focus on brivanib. Dig Dis 2011;29:316320.

48 Zhu AX, Sahani DV, Duda DG, di Tomaso E, Ancukiewicz M, Catalano OA, et al: Efficacy, safety, and potential biomarkers of sunitinib monotherapy in advanced hepatocellular carcinoma: a phase II study. J Clin Oncol 2009;27:3027-3035.

49 Cheng A, Kang Y, Lin D; PJ, Kudo M, Qin S, et al: Phase III trial of sunitinib (Su) versus sorafenib (So) in advanced hepatocellular carcinoma (HCC). J Clin Oncol 2011;29(suppl):4000. 
50 Y Arai YI, T Yamamoto, F Kanai, T Aramaki, T Tanaka, K Yamakado, M Kudo, S Kaneko, K Imanaka: A randomized phase II study of TSU-68 in patients (pts) with hepatocellular carcinoma (HCC) treated by transarterial chemoembolization (TACE). 2010 ASCO Annual Meeting; 2010; Chicago, IL: ASCO; 2010;p. 15s, abstract 4030 .

51 Kudo M: mTOR inhibitor for the treatment of hepatocellular carcinoma. Dig Dis 2011;29:310-315.

52 Zhu AX, Abrams TA, Miksad R, Blaszkowsky LS, Meyerhardt JA, Zheng H, et al: Phase 1/2 study of everolimus in advanced hepatocellular carcinoma. Cancer 2011;117:5094-5102.

53 Grünwald V, Merseburger AS: Axitinib for the treatment of patients with advanced metastatic renal cell carcinoma (mRCC) after failure of prior systemic treatment. Onco Targets Ther 2012;5:111-117.

54 Mancuso MR, Davis R, Norberg SM, O’Brien S, Sennino B, Nakahara T, et al: Rapid vascular regrowth in tumors after reversal of VEGF inhibition. J Clin Invest 2006;116:2610-2621.

55 Llovet JM, Pena CE, Lathia CD, Shan M, Meinhardt G, Bruix J: Plasma biomarkers as predictors of outcome in patients with advanced hepatocellular carcinoma. Clinical Cancer Research: an official journal of the American Association for Cancer Research. 2012;18:2290-2300.

56 Cabrera R, Pannu DS, Caridi J, Firpi RJ, Soldevila-Pico C, Morelli G, et al: The combination of sorafenib with transarterial chemoembolisation for hepatocellular carcinoma. Aliment Pharmacol Ther 2011;34:205213. 